\title{
The Misspecification of Expectations in New Keynesian Models: A DSGE-VAR Approach
}

\author{
Stephen J. COLE \\ Department of Economics \\ University of California, Irvine
}

\author{
Fabio MILANI* \\ Department of Economics \\ University of California, Irvine
}

April, 2014

\begin{abstract}
This paper tests the ability of popular New Keynesian models, which are traditionally used to study monetary policy and business cycles, to match the data regarding a key channel for monetary transmission: the dynamic interactions between macroeconomic variables and their corresponding expectations.

In the empirical analysis, we exploit direct data on expectations from surveys. To explain the joint evolution of realized variables and expectations, we adopt a DSGE-VAR approach, which allows us to estimate all models in the continuum between the extremes of an unrestricted VAR, on one side, and a DSGE model in which the cross-equation restrictions are dogmatically imposed, on the other side. Moreover, the DSGE-VAR approach allows us to assess the extent, as well as the main sources, of misspecification in the model.

The paper's results illustrate the failure of New Keynesian models under the rational expectations hypothesis to account for the dynamic interactions between observed macroeconomic expectations and macroeconomic realizations. Confirming previous studies, DSGE restrictions prove valuable when the New Keynesian model is exempted from matching observed expectations. But when the model is required to match data on expectations, it can do so only by moving away, and hence substantially rejecting, DSGE restrictions.

Finally, we investigate alternative models of expectations formation, including examples of extrapolative and heterogeneous expectations, and show that they can go some way toward reconciling the New Keynesian model with the data. Intermediate DSGE-VAR models, which avail themselves of DSGE prior restrictions, return to fit the data better than the unrestricted VAR. Hence, the results overall point to misspecification in the expectations formation side of the DSGE model, more than in the structural microfounded equations.
\end{abstract}

Keywords: Modeling of Expectations, DSGE Models, Rational Expectations, Observed Survey Expectations, Model Misspecification, DSGE-VAR, Heterogeneous Expectations.

JEL classification: C52, D84, E32, E50, E60.

* Corresponding Author: Fabio Milani, Department of Economics, 3151 Social Science Plaza, University of California, Irvine, CA 92697-5100. Phone: 949-824-4519. Fax: 949-824-2182. E-mail: fmilani@uci.edu. Homepage: http://www.socsci.uci.edu/ fmilani. 


\section{Introduction}

Expectations have always played a central role in models of monetary policy analysis. Fluctuations in real activity and inflation are in large part driven by expectations about future demand, inflation, and monetary and fiscal policy choices. Over the last two decades, the management of expectations has evolved into probably the most fundamental and widely discussed component of monetary policy-making. Woodford (2003), in his seminal textbook on New Keynesian thought, writes "For successful monetary policy is not so much a matter of effective control of overnight interest rates as it is of shaping market expectations of the way in which interest rates, inflation, and income are likely to evolve over the coming year and later." He then goes on to add that not only expectations matter in policy-making, but little else matters. Svensson (2004), Bernanke (2004), and Blinder et al (2008), as well as many other monetary researchers and policymakers, subscribe to the same view that recognizes the expectations channel as the main channel for stabilization policy.

In state-of-the-art monetary business cycle models, macroeconomic expectations are almost universally modeled according to the Rational Expectations Hypothesis (REH). Agents form modelconsistent expectations using all available information. They are assumed to know the correct model of the economy, its parameters, the distribution of the shocks, and so forth. Whereas various elements of the models are routinely checked and tested one against the other, the REH is often taken for granted in empirical work and left unchecked.

But are New Keynesian models subject to the REH able to capture the patterns of interactions between macroeconomic expectations and macroeconomic realizations that are observed in the data?

We argue that understanding the model's ability to match the data in this dimension is particularly critical in light of the fundamental role played by expectations, and the growing view of monetary policy as management of expectations. Furthermore, the focus on expectations has increased even more in the aftermath of the Great Recession, in light of current efforts by central banks around the world to provide markets with "forward guidance", with the aim of creating expectations of expansionary future monetary policies and thus stimulate their respective economies.

Our Approach. We exploit data on survey expectations to estimate a benchmark New Keynesian model with frictions and rational expectations. We use a DSGE-VAR approach to study misspecification in the model, with a particular focus on potential misspecification in the expectations block.

The DSGE-VAR approach, proposed by Del Negro and Schorfheide (2004), and also used in Del Negro and Schorfheide (2005, 2009) and Del Negro et al (2007), allows us to evaluate in the esti- 
mation all models in the continuum between two extremes: the rational expectations DSGE model, which imposes all the cross-equation restrictions that exist under the REH, and the unrestricted VAR model, which characterizes the joint dynamics of the observable variables and expectations without imposing restrictions from theory or the REH. A parameter, which will be denoted by $\lambda$, governs the weight placed on the DSGE restrictions in the specification that is found to be favored by the data within the model continuum. This approach is particularly suited to investigate misspecification of structural models. A similar approach has been used by Del Negro et al (2007) to identify the areas of misspecification in small and medium-scale DSGE models. As typical in the DSGE literature, however, they retain the assumption of rational expectations throughout the analysis. Here, we explicitly focus on potential misspecification in the way that expectation formation is modeled.

We start by estimating the New Keynesian model under the conventional assumption of rational expectations. We then include observed survey data to the set of observable variables that the estimation is required to match. We can, therefore, evaluate whether the DSGE's restrictions prove useful in successfully capturing the co-movement between macroeconomic expectations and outcomes, or, alternatively, whether the data suggest the rejection of such restrictions.

Results. The DSGE-VAR approach reveals a substantial failure of the New Keynesian model under rational expectations to match the joint evolution between macroeconomic observations and expectations that exists in the data.

The estimated prior tightness parameter $\lambda$ declines toward values favoring the unrestricted VAR, in the case in which observed expectations are added to the estimation. DSGE restrictions obtain a higher weight when the model with rational expectations is freed from the need to match actual data on expectations.

We then proceed to study misspecification in more detail by checking where exactly the model is failing. We do so by comparing the impulse responses obtained for the best-fitting DSGE-VAR model with the optimal $\lambda$ with those corresponding to the model in which the DSGE restrictions are imposed. The DSGE model's responses predominantly and continually leave the probability intervals implied by the DSGE-VAR model with the optimal $\lambda$. In various instances, the DSGE model's responses of output growth, inflation, and the interest rate, fail to match the required persistence found in the best-fitting DSGE-VAR model. The responses of expectations within the DSGE model are often misspecified and display signs and patterns inconsistent with the responses of survey expectations in the data.

We therefore propose a selection of alternative expectation formation schemes that relax, in part 
or fully, the REH. The first alternative is based on a perceived law of motion that has the same structural form as the minimum state variable solution of the model (excluding the disturbances, which are now assumed to remain outside the agents' information set), and that corresponds to a VAR model in output, inflation, and interest rates (we also consider a misspecified AR(1) law of motion, as alternative). This expectation formation assumption is in the spirit of learning models (Evans and Honkapohja, 2001, Milani, 2007); we do not, however, directly incorporate learning. The second deviation allows for heterogeneous expectations. We assume that a share of private-sector agents in the economy forms expectations according to the previously-described VAR model, while the remaining share forms rational expectations. Finally, as a third alternative, we motivate our expectation models from the evidence on expectation formation available from laboratory experiments. Hommes (2011) and Assenza et al (2012), show that expectations from the lab are clearly heterogeneous and cluster around three groups: adaptive expectations, trendfollowing expectations, and 'anchor and adjustment' rules. We insert those expectation formation rules in our New Keynesian model and add a share of agents maintaining rational expectations. The relative shares are estimated from the data. Besides changing the expectations formation rules, we also allow expectations to be affected by expectation shocks, which are independent from fundamentals, and potentially account for excesses of optimism and pessimism. The use of these expectation shocks are motivated by empirical evidence in Milani (2011, 2013).

We find that for the alternative expectation models, the overall fit substantially improves. Moreover, the DSGE-VAR estimations show that the model-imposed restrictions now become more valuable, since larger values of $\lambda$ provide the best fit of the data. The parsimonious cases with the $\mathrm{AR}$ and VAR perceived laws of motions are the overall best-fitting specification.

Expectation shocks are also important. For instance, an expectation shock to expected oneperiod ahead output growth is found to explain $86 \%, 28.1 \%$, and $41.7 \%$ of fluctuations in output growth, inflation, and the interest rate, respectively. The expectation shocks also explain a large amount of fluctuations in the expectation series of output growth and inflation $(53.9 \%$ and $46.8 \%)$. These shares collapse to zero by construction in benchmark rational expectation models.

Related literatures. The main scope of the paper is to highlight the misspecification of New Keynesian models of monetary policy in a key area: the empirical relationship between macroeconomic expectations and realizations. As we discussed, this misspecification is particularly relevant in light of recent studies investigating the potential impact of central bank communication, announcements, forward guidance policies, which heavily relies on private sector expectations responding as envisioned. Del Negro et al (2012) show that the impact of forward guidance in New Keynesian 
DSGE models is exceedingly large to be deemed believable. Our paper has a different focus, but it further stresses the failure of the model under rational expectations to capture the behavior of expectations.

Our use of a DSGE-VAR approach expands the literature in the direction of testing the REH. We add to previous studies (Del Negro and Schorfheide, 2004, 2005, 2009, Del Negro et al 2007), which highlight the promise of DSGE-VARs as means of investigating the sources of misspecification in empirical DSGE models, although always retaining the REH. Granziera (2012) also focuses on expectations formation, but she is interested in testing the empirical evidence of adaptive learning. She does not exploit data on survey expectations in the DSGE-VAR estimation.

Finally, the paper closely relates to the literatures that have focused on relaxing the rational expectations hypothesis. A large body of literature relaxes rational expectations to assume that agents are learning about the economy over time. The studies in this area study eventual convergence to the Rational Expectations Equilibrium (Evans and Honkapohja, 2001), the contribution of transitional learning to business cycles (Milani, 2007, 2011, Eusepi and Preston, 2011). Other studies emphasize the importance of heterogeneous expectations. Branch and McGough (2009) incorporate heterogeneous expectations in a New Keynesian model. Hommes (2011) provides experimental evidence on the importance of heterogeneity and adaptive behavior in the formation of expectations. We show in this paper that modeling expectations as suggested by learning or heterogeneous expectation approaches can considerably improve the fit of a benchmark New Keynesian model to the data.

\section{New Keynesian Model}

The following section describes the benchmark New Keynesian model that we use in the empirical analysis. The model is built from explicit microeconomic foundations and it includes the endogenous sources of persistence that are typically necessary to fit macroeconomic data, such as habit formation in private expenditures and indexation to lagged inflation in firms' price-setting. The model is thoroughly analyzed in Woodford (2003) and it has been estimated in Giannoni and Woodford (2004) and Milani (2007), among others. ${ }^{1}$ The log-linearized aggregate demand, Phillips

\footnotetext{
${ }^{1}$ Medium-scale and large-scale DSGE models expand on the current framework by adding capital accumulation, investment decisions, variable capacity utilization, imperfectly competitive labor markets, and a variety of additional features.
} 
curve, and monetary policy equations are given by

$$
\begin{aligned}
\tilde{x}_{t} & =E_{t} \tilde{x}_{t+1}-(1-\beta \eta) \sigma\left[i_{t}-E_{t} \pi_{t+1}-r_{t}^{n}\right] \\
\tilde{\pi}_{t} & =\xi_{p}\left[\omega x_{t}+[(1-\eta \beta) \sigma]^{-1} \tilde{x}_{t}\right]+\beta E_{t} \tilde{\pi}_{t+1}+\mu_{t} \\
i_{t} & =\rho i_{t-1}+(1-\rho)\left[\chi_{\pi} \pi_{t}+\chi_{x} x_{t}\right]+\varepsilon_{t}^{m p}
\end{aligned}
$$

where

$$
\begin{aligned}
\tilde{\pi}_{t} & \equiv \pi_{t}-\gamma \pi_{t-1} \\
\tilde{x}_{t} & \equiv\left(x_{t}-\eta x_{t-1}\right)-\beta \eta E_{t}\left(x_{t+1}-\eta x_{t}\right),
\end{aligned}
$$

and where $x_{t}, \pi_{t}$, and $i_{t}$ denote the output gap, inflation, and the nominal interest rate, respectively. Equation (1) describes aggregate demand: the current gap between actual and potential output is a function of expected one-period and two-period ahead output gaps, expected one-period ahead inflation, the nominal interest rate, and the natural real interest rate disturbance $r_{t}^{n}$. Households' preferences are characterized by internal habit formation, with the coefficient $0 \leq \eta \leq 1$ denoting the strength of habits in their utility function. The household's discount rate is given by $0 \leq \beta \leq 1$, whereas $\sigma>0$ measures the elasticity of intertemporal substitution of consumption. Equation (2) is the New Keynesian Phillips curve, which expresses inflation as a function of lagged, current, and expected one-period ahead output gaps, lagged and expected one-period ahead inflation, and a cost-push shock $\mu_{t}$. This equation derives from the profit maximization decision of monopolistically competitive firms. Following the price-setting framework of Calvo (1983), a fraction $\theta$ of firms are not able to re-optimize each period. The composite coefficient $\xi_{p} \equiv(1-\theta \beta)(1-\theta) / \theta$ is inversely related to the degree of price stickiness in the economy. The fraction of firms not re-optimizing index price to past inflation following Christiano et al (2005), with $0 \leq \gamma \leq 1$ denoting the degree of inflation indexation. The elasticity of marginal costs with respect to output is defined by the parameter $\omega$. Finally, equation (3) characterizes monetary policy in the economy as a Taylor rule with interest-rate smoothing. The central bank adjusts the nominal interest rate based on the lagged nominal interest rate, current inflation and output gap; $\varepsilon_{t}^{m p}$ captures unanticipated monetary policy shocks. The feedback coefficients $\chi_{\pi}$ and $\chi_{x}$ denote the weights place on the control of inflation and the output gap. The parameter $\rho$ captures the central bank preference for smoothing interest rate changes over time.

The structural disturbances, with the exception of the monetary policy shock, which is conven- 
tionally modeled as i.i.d., evolve as $\mathrm{AR}(1)$ processes:

$$
\begin{aligned}
r_{t}^{n} & =\phi_{d} r_{t-1}^{n}+\sigma_{r} \varepsilon_{t}^{r} \\
\mu_{t} & =\phi_{\mu} \mu_{t-1}+\sigma_{\mu} \varepsilon_{t}^{\mu} .
\end{aligned}
$$

All innovations $\varepsilon_{t}^{m p}, \varepsilon_{t}^{r}$, and $\varepsilon_{t}^{\mu}$, are assumed to be drawn from a Normal distribution.

Following the dominant paradigm in macroeconomics, expectations, denoted by the mathematical operator $E_{t}$, are modeled for now according to the rational expectation hypothesis.

\subsection{RE Solution and State-Space Representation}

The structural model can be rewritten in state-space form as:

$$
\Gamma_{0} X_{t}=\Gamma_{1} X_{t-1}+\Pi \epsilon_{t}+\Psi \eta_{t},
$$

where $X_{t}$ includes the endogenous variables, the expectation terms, and the AR disturbances, $\epsilon_{t}$ is a vector including the model's exogenous innovations, and $\eta_{t}=X_{t}-E_{t-1} X_{t}$ represents a vector of expectational errors, such that $E_{t} \eta_{t+1}=0$. The model written in this form can be solved under the assumption of rational expectations, using the approach laid out in Sims (2000). Under rational expectations, the expectational errors disappear from the model as they are mapped into the set of structural shocks. The solution can be written in state space as

$$
X_{t}=F X_{t-1}+G \epsilon_{t},
$$

which yields the transition equation for our state space model.

We will estimate the model both omitting and including the available data series on expectations as observables, in addition to macroeconomic realizations on output growth, inflation, and the interest rate.

The transition equation is, therefore, augmented with the following set of observation equations, expressed here for the case in which expectations series are added to the list of observables:

$$
\left[\begin{array}{c}
g_{t}^{o b s} \\
\pi_{t}^{o b s} \\
i_{t}^{o b s} \\
\mathbb{E}_{t}^{o b s} g_{t+1} \\
\mathbb{E}_{t}^{o b s} g_{t+2} \\
\mathbb{E}_{t}^{o b s} \pi_{t+1}
\end{array}\right]=\left[\begin{array}{c}
g_{t} \\
\pi_{t} \\
i_{t} \\
E_{t} g_{t+1} \\
E_{t} g_{t+2} \\
E_{t} \pi_{t+1}
\end{array}\right]+\left[\begin{array}{c}
\mathbf{0}_{3 \times 3} \\
\mathbf{I}_{3 \times 3}
\end{array}\right]\left[\begin{array}{c}
o_{t}^{g_{t+1}} \\
o_{t}^{g_{t+2}} \\
o_{t}
\end{array}\right]
$$

where $g_{t}$ denotes the growth rate of real output, and $E_{t} g_{t+1}, E_{t} g_{t+2}$, denote one-period-ahead and two-period-ahead real output growth expectations. Therefore, we assume that observed expectations are equal to the rational expectations implied by the model plus a i.i.d measurement error, denoted by $o_{t}$. 


\section{DSGE-VAR Estimation}

\subsection{The DSGE-VAR Approach}

The DSGE-VAR estimation approach used in this paper follows the framework developed in Del Negro and Schorfheide (2004). We provide some intuition first and lay out the technical details later. The DSGE-VAR method exploits information from a DSGE model to aid in estimating the parameters of a VAR. While VARs are typically successful in identifying interrelationships among variables under minimal theoretical restrictions, and outperform various alternatives in out-ofsample forecasting, one of their most significant drawbacks is that they are far from parsimonious. The DSGE-VAR approach helps in this dimension by essentially shrinking the VAR parameter subspace toward the values implied by the DSGE model. At the same time, the approach improves over structural DSGE estimation by providing it with more flexibility: the DSGE cross-equation restrictions may not necessarily be imposed dogmatically, but the data are allowed to deviate from them (or from some of them) to the extent they need.

To aid the interpretation, one way to think about the DSGE-VAR approach is as follows. For a given value of the DSGE model's parameters, and given realizations of the shocks, the DSGE model is simulated to generate artificial data. Adopting a DSGE model prior, in fact, is equivalent to augmenting the data with new, generated, dummy observations. The parameters of the VAR model are subsequently estimated using a sample merging the DSGE simulated data and real data. A key parameter, which will be denoted by $\lambda$, can be interpreted, for now, as the ratio of artificial DSGE observations over actual observations. If the process is repeated for different values of the DSGE parameters, and if the DSGE is covariance-stationary, a mapping can be defined between the VAR and DSGE parameters. The mapping creates a restriction function for the VAR parameters based on the DSGE model. If $\lambda \rightarrow 0$, the DSGE contains no useful information: the best fit is obtained when the artificial DSGE observations and, hence, their implied theoretical restrictions, are entirely ignored. If $\lambda \rightarrow \infty$, DSGE prior dummy observation dominate the sample. Therefore, the DSGE model provides a superior description of the data. The parameter $\lambda$, therefore, can measure the relative fit of the DSGE model to the VAR model.

A key parameter, which will be denoted by $\lambda$, can be interpreted, for now, as the ratio of artificial DSGE observations over actual observations. If $\lambda \rightarrow 0$, the DSGE contains no useful information: the best fit is obtained when the artificial DSGE observations and, hence, their implied theoretical restrictions, are entirely ignored. If $\lambda \rightarrow \infty$, DSGE prior dummy observation dominate the sample. Therefore, the DSGE model provides a superior description of the data. The parameter $\lambda$, therefore, can measure the relative fit of the DSGE model to the VAR model. 
In practice, $\lambda$ will scale the standard deviation of the variance-covariance matrix of the priors for the VAR coefficients. The DSGE-VAR estimation considers all possible cases in the continuum between the VAR and the DSGE model. A small $\lambda$ indicates that the VAR coefficient prior distributions are centered at the values consistent with DSGE restrictions, but they are extremely diffuse; a large $\lambda$ indicates a prior that is more tightly centered around the DSGE restrictions (with a lower variance). By finding the best-fitting $\lambda$, we can gain intuition about how useful DSGE restrictions are in explaining the data.

To describe the procedure in more technical terms, let's start from a typical VAR model with $p$ lags: ${ }^{2}$

$$
y_{t}=\phi_{0}+\sum_{i=1}^{p} \phi_{i} y_{t-i}+\epsilon_{t}
$$

where $y_{t}$ is an $n \times 1$ vector of endogenous variables and $\epsilon_{t}$ denotes the error term, distributed as $N\left(0, \Sigma_{\epsilon}\right)$. The VAR can be rewritten as

$$
Y=X \Phi+\Xi
$$

where $Y$ is a $T \times n$ matrix with rows given by $y_{t}^{\prime}, t=1, \ldots, T, X$ is a $T \times k$ matrix, where $k=1+n p$, and with rows $x_{t}^{\prime}=\left[1, y_{t-1}^{\prime}, \ldots, y_{t-p}^{\prime}\right], \Phi=\left[\phi_{0}, \phi_{1}, \ldots, \phi_{p}\right]^{\prime}$, and $\Xi$ is a $T \times n$ matrix that has rows $\epsilon_{t}^{\prime}$.

The VAR likelihood function is, therefore, given by the familiar:

$$
p\left(Y \mid \Phi, \Sigma_{\epsilon}\right) \propto\left|\Sigma_{\epsilon}\right|^{\frac{-T}{2}} \exp \left\{-\left(\frac{1}{2}\right) \operatorname{tr}\left[\Sigma_{\epsilon}^{-1}\left(Y^{\prime} Y-\Phi^{\prime} X^{\prime} Y-Y^{\prime} X \Phi+\Phi^{\prime} X^{\prime} X \Phi\right)\right]\right\} .
$$

In a DSGE-VAR, the priors for the VAR coefficients $\Phi$ and $\Sigma_{\epsilon}$, conditional on the DSGE parameter vector $\theta$, are obtained as if a set of $T^{*}=\lambda T$ simulated data are generated from the DSGE model and combined with the actual observations $T$ in the estimation. Therefore, the likelihood for the combined sample, including both the $T^{*}$ artificially-generated observations from the DSGE model $\left(Y^{*}(\theta), X^{*}(\theta)\right)$, and the actual $T$ sample observations, can be computed by multiplying

$$
p\left(Y^{*}(\theta) \mid \Phi, \Sigma_{\epsilon}\right) \propto\left|\Sigma_{\epsilon}\right|^{\frac{-\lambda T}{2}} \exp \left\{-\left(\frac{1}{2}\right) \operatorname{tr}\left[\Sigma_{\epsilon}^{-1}\left(Y^{* \prime} Y^{*}-\Phi^{\prime} X^{* \prime} Y^{*}-Y^{* \prime} X^{*} \Phi+\Phi^{\prime} X^{* \prime} X^{*} \Phi\right)\right]\right\}
$$

with $p\left(Y \mid \Phi, \Sigma_{\epsilon}\right)$ as given by expression (11).

Instead of actually generating the artificial data set $\left(Y^{*}, X^{*}\right)$ and using the sample moments $Y^{* \prime} Y^{*}, X^{* \prime} Y^{*}, Y^{* \prime} X^{*}, X^{* \prime} X^{*}$, however, if $y_{t}$ is covariance stationary as implied by the DSGE model, we can replace them with the scaled population moments $\lambda T \Gamma_{y y}^{*}(\theta)=E_{\theta}\left[y_{t} y_{t}^{\prime}\right], \lambda T \Gamma_{y x}^{*}(\theta)=$ $E_{\theta}\left[y_{t} x_{t}^{\prime}\right], \lambda T \Gamma_{x y}^{*}(\theta)=E_{\theta}\left[x_{t} y_{t}^{\prime}\right]$, and $\lambda T \Gamma_{x x}^{*}(\theta)=E_{\theta}\left[x_{t} x_{t}^{\prime}\right]$. Conditional on the DSGE parameter

\footnotetext{
${ }^{2}$ We refer readers to Del Negro and Schorfheide (2004) for full details on the procedure.
} 
vector $\theta$, such moments can be obtained analytically, substantially reducing the computational effort.

Thus, with the inclusion of an initial improper prior $p\left(\Phi, \Sigma_{\epsilon}\right) \propto\left|\Sigma_{\epsilon}\right|^{\frac{-(n+1)}{2}}$, equation (12) becomes

$$
\begin{aligned}
p\left(\Phi, \Sigma_{\epsilon} \mid \theta\right) & =c^{-1}(\theta)\left|\Sigma_{\epsilon}\right| \frac{-(\lambda T+n+1)}{2} \exp \left\{-\left(\frac{1}{2}\right) \operatorname{tr}\left[\lambda T \Sigma _ { \epsilon } ^ { - 1 } \left(\Gamma_{y y}^{*}(\theta)\right.\right.\right. \\
& \left.\left.\left.-\Phi^{\prime} \Gamma_{x y}^{*}(\theta)-\Gamma_{y x}^{*}(\theta) \Phi+\Phi^{\prime} \Gamma_{x x}^{*}(\theta) \Phi\right)\right]\right\},
\end{aligned}
$$

where $c^{-1}(\theta)$ is a normalizing constant, obtained so that the density in (13) integrates to one. If $\lambda T \geq k+n$ and $\Gamma_{x x}(\theta)$ is invertible, equation (13) is proper, and $c(\theta)$ is defined as

$$
c(\theta)=(2 \pi)^{\frac{n k}{2}}\left|\lambda T \Gamma_{x x}^{*}(\theta)\right|^{\frac{-n}{2}}\left|\lambda T \Sigma_{\epsilon}^{*}(\theta)\right|^{\frac{-(\lambda T-k)}{2}} 2^{\frac{n(\lambda T-k)}{2}} \pi^{\frac{n(n-1)}{4}} \prod_{i=1}^{n} \Gamma[(\lambda T-k+1-i) / 2],
$$

where $\Gamma(\cdot)$ indicates the Gamma function. Conditioning on the DSGE parameters $\theta$, the prior distribution (13) for the VAR parameters belongs to the Normal - Inverse Wishart class:

$$
\begin{array}{r}
\Phi \mid \Sigma_{\epsilon}, \theta, \lambda \sim N\left(\Phi^{*}(\theta), \Sigma_{\epsilon} \otimes\left(\lambda T \Gamma_{x x}^{*}(\theta)\right)^{-1}\right) \\
\Sigma_{\epsilon} \mid \theta, \lambda \sim I W\left(\lambda T \Sigma_{\epsilon}^{*}(\theta), \lambda T-k, n\right),
\end{array}
$$

where $\Phi^{*}(\theta)=\Gamma_{x x}{ }^{*-1}(\theta) \Gamma_{x y}^{*}(\theta)$ and $\Sigma_{\epsilon}^{*}(\theta)=\Gamma_{y y}^{*}(\theta)-\Gamma_{y x}^{*}(\theta) \Gamma_{x x}^{*-1}(\theta) \Gamma_{x y}{ }^{*}(\theta)$. In our procedure, we also define $\lambda$ as a parameter to be estimated. Thus, our DSGE-VAR model is modified to include a prior for $\lambda$. The new prior, which is independent from $\theta$, takes the form

$$
p\left(\Phi, \Sigma_{\epsilon}, \theta, \lambda\right)=p(\Phi, \Sigma \mid \theta, \lambda) p(\theta) p(\lambda)
$$

The posterior distribution can be rewritten

$$
p\left(\Phi, \Sigma_{\epsilon}, \theta \mid Y\right)=p\left(\Phi, \Sigma_{\epsilon} \mid Y, \theta\right) p(\theta, \lambda \mid Y) .
$$

We can find an expression for $p\left(\Phi, \Sigma_{\epsilon} \mid Y, \theta\right)$. By noting that equations (15) and (16) define a conjugate prior for $p\left(\Phi, \Sigma_{\epsilon} \mid Y, \theta\right)$, we see that the VAR posterior distribution $p\left(\Phi, \Sigma_{\epsilon} \mid Y, \theta\right)$ is from the same family of distributions. Thus, the posterior distributions of $\Phi$ and $\Sigma_{\epsilon}$ are defined as

$$
\begin{gathered}
\Phi \mid Y, \Sigma_{\epsilon}, \theta \sim N\left(\tilde{\Phi}(\theta), \Sigma_{\epsilon} \otimes\left(\lambda T \Gamma_{x x}^{*}(\theta)+X^{\prime} X\right)^{-1}\right) \\
\Sigma_{\epsilon} \mid Y, \theta \sim I W\left((\lambda+1) T \tilde{\Sigma}_{\epsilon}(\theta),(1+\lambda) T-k, n\right),
\end{gathered}
$$

where

$$
\tilde{\Phi}(\theta)=\left(\lambda T \Gamma_{x x}^{*}(\theta)+X^{\prime} X\right)^{-1}\left(\lambda T \Gamma_{x y}^{*}+X^{\prime} Y\right)
$$




$$
\begin{aligned}
\tilde{\Sigma}_{\epsilon}(\theta) & =\frac{1}{(\lambda+1) T}\left[\left(\lambda T \Gamma_{y y}^{*}(\theta)+Y^{\prime} Y\right)-\left(\lambda T \Gamma_{y x}^{*}(\theta)\right.\right. \\
& \left.\left.+Y^{\prime} X\right)\left(\lambda T \Gamma_{x x}^{*}(\theta)+X^{\prime} X\right)^{-1}\left(\lambda T \Gamma_{x y}^{*}(\theta)+X^{\prime} Y\right)\right]
\end{aligned}
$$

can be interpreted as Maximum Likelihood estimates of $\Phi$ and $\Sigma_{\epsilon}$. The last term in equation (18), $p(\theta, \lambda \mid Y)$, does not have a closed form solution, but we use a Random Walk Metropolis-Hastings algorithm, similar to the one described in Del Negro and Schorfheide (2004), to sample values of $\theta$ and $\lambda$ from the posterior distribution.

\subsection{Data description}

We use quarterly data on real GDP growth, GDP implicit price deflator inflation, and the Federal funds rate, from FRED, as observable variables that need to be matched in the estimation. For estimated DSGE and DSGE-VAR models with expectations, we add to the previous set of realized series observed data on expectations, which enter the measurement equation as shown in (8). We use expectations about one-period-ahead real GDP growth, two-period-ahead real GDP growth, and one-period-ahead GDP deflator inflation. These are the expectations that directly enter the model and are obtained from the Survey of Professional Forecasters (we use means across forecasters). ${ }^{3}$ The sample in the estimation spans the years between the last quarter of 1968, chosen because the survey series on expectations start from this date, and 2009, when we stop to avoid the nonlinearity imposed by the binding zero-lower-bound constraint in the subsequent years.

Figure 1 shows the relation over the sample among realizations for output growth and inflation and the corresponding expectations series from the SPF.

\subsection{Prior Selection and Bayesian Estimation}

Our choices for the parameters' prior distributions are shown in Table 1. We select a Gamma prior for the intertemporal elasticity $\sigma$ with mean equal to 1 . The priors for $\xi_{p}$ and $\omega$ have means that are chosen based on Giannoni and Woodford (2004). We use inverse gamma priors for the standard deviation coefficients of the structural innovations. For the standard deviations related to measurement error, instead, we use Gamma(0.3,0.3) distributions, which allow us to assign higher probability to values that fall closer to zero than to larger values. We choose Beta distributions with mean 0.7 for the habit formation in consumption coefficient and with mean 0.5 for the inflation indexation coefficient. Finally, a key parameter of focus in the paper is $\lambda$, indicating the tightness of DSGE prior restrictions. We use an uninformative uniform prior between the allowed $\lambda_{\text {min }}$ and

\footnotetext{
${ }^{3}$ The acronyms for the series we use are GDPC1, GDPDEF, and FEDFUNDS, for real GDP, GDP deflator, and Federal funds rate, from FRED, and NGDP, PGDP, to construct the implied one-period-ahead and two-periodahead forecasts for real GDP growth and one-period-ahead forecasts for inflation, from the Survey of Professional Forecasters.
} 
$\infty .{ }^{4}$ We have experimented with more informative Gamma priors and, given that the data appear very informative, the results were entirely similar.

We estimate the combined DSGE-VAR models using Bayesian methods. We generate draws through a Metropolis-Hastings algorithm. We run four chains of one million draws each, starting from different initial values and discarding a burn-in of 200,000 draws. To choose the ideal lag length, we performed a search by estimating all DSGE-VAR models with lag length going from one to eight. For each estimated DSGE-VAR, we report the results corresponding to the best-fitting specification. $^{5}$

\section{Empirical Results}

\subsection{RE without Expectations Data}

As a benchmark, we first estimate the New Keynesian model summarized by equations (1) to (5), with expectations formed according to the rational expectations hypothesis. We follow the conventional approach in the empirical macroeconomic literature by not requiring rational expectations to try to fit the corresponding observed expectations from surveys.

Table 1 shows the posterior results. The posterior mean estimates are in line with previous existing evidence. The model requires large degrees of habit formation $(\eta=0.74)$ and inflation indexation $(\gamma=0.50)$ to match the persistence in the data. The disturbance entering the aggregate demand equation displays a sizable serial correlation, with a posterior mean for the AR coefficient equal to 0.84 . We obtain moderate degrees of intertemporal substitution $(\sigma=0.67)$ and a slope of the Phillips curve coefficient $\xi_{p}$ equal to 0.0014 , the latter in line with the estimate in Giannoni and Woodford (2004). ${ }^{6}$

The main interest in the estimation, however, lies in the estimate of the best-fitting $\lambda$, governing the tightness of the DSGE prior restrictions that are imposed on the VAR coefficients. We obtain a value of $\lambda$ equal to 0.57 . To facilitate comparison across different estimations, we also present a relative indicator of distance between the DSGE and VAR specifications, which corrects for the different values of $\lambda_{\text {min }}$. The relative indicator, as suggested by Adolfson et al (2008) is given by $\left(\lambda_{o p t}-\lambda_{\min }\right)$ and, here, it is equal to 0.43 . The results, therefore, conform with the evidence in Del Negro and Schorfheide (2004) and Del Negro et al (2007): a DSGE-VAR with intermediate $\lambda$ fits the data best, outperforming both the DSGE and the VAR benchmarks. The DSGE restrictions

\footnotetext{
${ }^{4}$ The lower bound $\lambda_{\min }$ is given by $\lambda_{\min }=(n+k) / T$, where $k=1+p n$, and where $n$ denotes the number of endogenous variables, $p$ the number of lags, and $T$ the number of time series observations.

${ }^{5}$ The resulting best-fitting specifications have two lags for the DSGE-VAR with rational expectations and survey expectations as observables, and six lags for the other cases.

${ }^{6}$ The data, however, are not very informative on the values of $\xi_{p}$ and $\omega$.
} 
are, therefore, useful in improving the unrestricted VAR estimates.

In this estimation scenario, however, we have let expectations free to adjust to fit the realized data. But do actual private-sector expectations behave in the same way as the implied rational expectations from the model? We now turn to investigate this issue by explicitly incorporating the available data on expectations from the Survey of Professional Forecasters in the estimation.

\subsection{RE with Expectations Data}

We re-estimate the model, but now adding observed survey expectations to the list of observable variables to match. Observed expectations and rational expectations obtained from the model are related through the measurement equations laid out in (8).

First, we re-estimate the DSGE-VAR to find the best-fitting specification in the model space between the unrestricted VAR and the fully-restricted DSGE model, i.e. the optimal $\lambda$. We then re-estimate the model by imposing the DSGE restrictions, i.e., we consider the $\operatorname{DSGE-\operatorname {VAR}}(\lambda=\infty)$ approximation. Estimation of these two cases allows us to study where misspecification takes place in the model. We can gain intuition on the areas of misspecification, for example, by comparing the impulse responses between the specification that yields the best fit of the data and the specification that dogmatically imposes the DSGE restrictions.

Table 1 shows the posterior estimates corresponding to the two cases. While the addition of expectations does not substantially alter the DSGE-VAR coefficient estimates, the conclusions regarding the ability of the DSGE restriction to help in explaining the data need to be largely reassessed.

When the estimation is required to match the co-movement observed in the data between macroeconomic outcomes and expectations, the data point toward substantially rejecting the DSGE restrictions. The posterior estimate for $\lambda$ is equal to 0.16 , only marginally larger than the minimum

allowed $\lambda$ (which falls around 0.08). Our relative indicator of misspecification $\left(\lambda_{\text {opt }}-\lambda_{\text {min }}\right)$, hence, falls from 0.43 to 0.08 , when the model is asked to match expectations. The DSGE model, therefore, is unable to capture the dynamic adjustment between macroeconomic data and expectations. We believe that this is a key failure of the New Keynesian model, given the role that the expectations channel plays both in the propagation of business cycles and in the transmission of monetary policy decisions to the economy.

Therefore, we seek to investigate in more depth where the main sources of misspecification in the theoretical model lie. Figures 2, 3, and 4, show the impulse response functions of output growth, inflation, the nominal interest rate, expected output growth, and expected inflation, to demand, 
supply, and monetary policy shocks. The DSGE model's impulse responses are compared to the impulse responses of the best-fitting DSGE-VAR with $\lambda=\lambda^{\hat{o p t}}$, which, as we have seen, is very close to an unrestricted VAR.

We summarize the main findings into three key points. First, the impulse responses confirm that the DSGE model is fundamentally misspecified and is unable to capture the dynamic movements found by the DSGE-VAR $\left(\lambda=\lambda^{\hat{o p t}}\right)$. The DSGE model's impulse responses continually leave the $95 \%$ probability bands implied by the $\operatorname{DSGE}-\operatorname{VAR}\left(\lambda=\lambda^{\hat{o p t}}\right)$ model. The exceptions are the responses of inflation and expected inflation to a monetary policy shock, which fall, however, around zero and display sizable uncertainty. In addition, the second finding concerns the persistence in the responses to the structural shocks. In particular, the DSGE model's response of inflation and interest rate to a cost-push shock does not exhibit as much persistence as the impulse responses from the $\operatorname{DSGE}-\operatorname{VAR}\left(\lambda=\lambda^{\hat{o p t}}\right)$ model. The third finding concerns the responses of the observed expectations to the structural shocks. The responses of expected inflation to a natural rate and costpush shock do not show enough persistence under the DSGE model. The DSGE-VAR $\left(\lambda=\lambda^{\hat{o p t}}\right)$ model exhibits a hump-shaped response, while the DSGE model quickly levels out. In the case of cost-push and monetary policy shocks, the DSGE model's impulse responses seem to follow different paths than those implied by the $\operatorname{DSGE}-\operatorname{VAR}\left(\lambda=\lambda^{\hat{o p t}}\right)$ model. Expected output growth (one and two-quarters ahead), displays responses that are very different in the DSGE model compared with the best-fitting DSGE-VAR benchmark, and that often have the opposite sign.

\section{Relaxing Rational Expectations: Alternative Expectation Formation Mechanisms}

To study whether rational expectations are responsible for the failure of the DSGE model to match the interactions between macroeconomic expectations and the corresponding realizations, we investigate the performance of the model under alternative expectation formation mechanisms.

\subsection{VAR \& AR Perceived Laws of Motion}

First, we assume that agents form expectations from a VAR, whose set of endogenous variables correspond to those that would appear in the MSV solution of the DSGE model under RE, and under the assumption, which we find more empirically realistic, that agents cannot observe the exogenous structural shocks. This corresponds to a specification that is usually chosen as a perceived law of motion in adaptive learning models. For simplicity, however, we do not allow here for learning. Adding time-varying coefficients to the DSGE-VAR framework is beyond the scope of 
this analysis. A study along these lines is Granziera (2012).

The VAR expectations are formed as

$$
\widehat{E}_{t-1} Y_{t+1}^{V A R}=a(1+b)+b^{2} Y_{t-1}+e_{t-1, t+1}
$$

where $Y_{t}=\left[x_{t}, \pi_{t}, i_{t}\right]^{\prime}$. To be consistent with the learning literature, we assume that economic agents observe only data up to $t-1$ when forming expectations in period $t$ about variables in $t+1$ and further. We also find that this case provides a better fit of the data than the time- $t$ information alternative. Moreover, a second important modification to the RE case is that we allow disturbances to affect the formation of expectations. We allow expectations to depart from the point VAR forecast by including an exogenous term, $e_{t-1, t+1}$, which can be interpreted as an expectation shock (the shock affects expectations related to $t+1$ variables, formed using $t-1$ information). The shock is in the spirit of the judgment variable studied in Bullard, Evans, and Honkapohja (2008), and of the expectation, or sentiment, shocks proposed in Milani $(2011,2013)$, who finds that they explain roughly half of business cycle fluctuations in general equilibrium settings.

As a second, related, specification, we simplify the previous case by assuming that agents form expectations from simpler AR(1) models for output and inflation. The expectations, denoted by $\widehat{E}_{t-1} Y_{t+1}^{A R}$, are formed as in (23), but now with $Y_{t}=\left[x_{t}, \pi_{t}\right]^{\prime}$ and with $b$ being a diagonal matrix.

\subsection{Heterogeneous Expectations}

Besides the expectation models motivated by the adaptive learning literature, we also examine alternatives that allow for heterogeneous expectations. A fraction $\omega_{V A R}$ of the agents in the model are assumed to form VAR expectations, as in the previous section, whereas the remaining fraction, $1-\omega_{V A R}$, forms rational expectations:

$$
\widehat{E}_{t} Y_{t+1}^{H e t}=\omega_{V A R} \widehat{E}_{t-1} Y_{t+1}^{V A R}+\left(1-\omega_{V A R}\right) E_{t} Y_{t+1}+e_{t-1, t+1}
$$

where $E_{t} Y_{t+1}$ denotes rational expectations, and where we allow, as in the previous section, for

an exogenous sentiment disturbance $e_{t-1, t+1}$ to affect aggregate expectations formation. In the estimation, we select an uninformative Uniform $(0,1)$ prior for the share parameter $\omega_{V A R}$.

\section{3 (Heterogeneous) Expectations from the Lab}

Hommes (2011) provides experimental evidence on the formation of macroeconomic expectations in a laboratory setting. Subjects in the experiment are asked to forecast future output and inflation, in an environment where actual data are generated by simulating a textbook New Keynesian model. 
Their main findings are that expectations are heterogeneous, but they seem to cluster around representative groups, who base their forecasts on simple heuristic rules. One group is characterized by trend-following expectations, another by adaptive expectations, and the last one by expectations that can be labeled as "anchor and adjustment". In this section, we let expectations in the model match the evidence from the lab, by allowing for heterogeneity and modeling expectations according to the clusters that emerged from the experiments.

The trend-following ( $\mathrm{TF}$ ) expectations, adaptive (AD), and the anchor and adjustment (AA) expectations are formed, respectively, as

$$
\begin{aligned}
\widehat{E}_{t}^{T F} Y_{t+1} & =Y_{t-1}+a\left(Y_{t-1}-Y_{t-2}\right) \\
\widehat{E}_{t}^{A D} Y_{t+1} & =b Y_{t-1}+(1-b) \widehat{E}_{t-1} Y_{t} \\
\widehat{E}_{t}^{A A} Y_{t+1} & =c\left(Y_{t-1}^{\text {ave }}+Y_{t-1}\right) / 2+\left(Y_{t-1}-Y_{t-2}\right)
\end{aligned}
$$

where $a, b, c$ are coefficients to be estimated and where $Y_{t-1}^{a v e}$, the anchor in the anchor and adjustment case, is computed as $Y_{t-1}^{a v e}=(1 / 8) \sum_{i=1}^{8} Y_{t-i}$.

We consider these expectation clusters in an encompassing model with heterogeneous expectations, which also includes a residual fraction of agents, who form rational expectations. Aggregate expectations equal

$$
\begin{aligned}
\widehat{E}_{t} Y_{t+1}= & \omega_{T F} \widehat{E}_{t}^{T F} Y_{t+1}+\omega_{A D} \widehat{E}_{t}^{A D} Y_{t+1}+\omega_{A A} \widehat{E}_{t}^{A A} Y_{t+1}+ \\
& \left(1-\omega_{T F}-\omega_{A D}-\omega_{A A}\right) E_{t} Y_{t+1}+d_{i} i_{t}+e_{t, t+1}
\end{aligned}
$$

In addition to the heterogeneous expectations formed as in Hommes (2011) and Assenza et al (2012), we add two modifications. First, to guarantee determinacy in the system, we allow expectations to respond to monetary policy decisions by adding the nominal interest rate in (28). ${ }^{7}$ Second, as in the previous cases, we allow agents to depart from the chosen models' point forecasts by including the aggregate expectation shock $e_{t, t+1}$.

In the estimation, we assume Beta prior distributions, with mean 0.167 and standard deviation 0.1 , for the $\omega_{T F, A D, A A}$ parameters. We select a $\mathrm{N}(0.4,0.2)$ prior for coefficients $a$, a Beta with mean 0.5 and standard deviation 0.2 for $b$, and a $\mathrm{N}(0.25,0.125)$ for $c$.

\subsection{Results Under Alternative Expectations}

We report the posterior estimates for the structural coefficients as well as for the optimal $\lambda$ in Table 2 under the alternative expectations. The coefficient estimates do not substantially vary from the

\footnotetext{
${ }^{7}$ This wasn't necessary in the heterogeneous expectation framework of the previous subsection, since VAR expectations already included a response to the interest rate.
} 
estimates presented in Table 1. The exception, however, is given by the optimal $\lambda$. The estimate for the optimal $\lambda$ is approximately 0.80 across the alternative expectations models. By allowing agents to have one of the alternative expectations, the DSGE model-imposed restrictions now become more valuable. The model fits the data better under the alternative expectation formation schemes. For any alternative expectations model, the estimated marginal likelihood substantially improves, reaching values between -189.46 and -195.33 , compared to the marginal likelihood for the model with rational expectations, which is equal to -215.49 .

The measures of relative fit $\left(\lambda_{\text {opt }}-\lambda_{\min }\right)$ equal to $0.53-0.54$, substantially rising from the value of 0.08 , obtained for the model that imposed fully-rational expectations. The single best-fitting model is the one assuming AR expectations, possibly because of its relative parsimony compared with the other models.

Evidence on the difficulties of rational expectations is also apparent looking at posterior estimates of the relative weights of boundedly-rational expectations. In the heterogeneous expectations model, the posterior means for the shares of VAR-based expectations equal 0.62 for output growth and 0.86 for inflation; rational expectations account only for the remaining part. In the alternative heterogeneous expectations model motivated by lab evidence, the posterior means for backwardlooking lab-based expectations sum to 0.60 for output growth and to 0.79 for inflation. ${ }^{8}$

\subsection{Variance Decomposition}

We also examine the merits of the DSGE model's restrictions by looking at the results of the forecast error variance decomposition displayed in Table 3. When allowing agents to form expectations from a small-scale VAR model, the expectational shocks seem to contribute a significant share to the forecast error variance in output growth, inflation, interest rate, expected output growth, and expected inflation. This result shows that the models with rational expectations fail to incorporate key drivers of fluctuations. Under the standard DSGE model with rational expectations, the main factor driving movements in output growth is the demand shock. The demand shock contributes $93.6 \%$ of the forecast error variance of output growth. When relaxing the DSGE restrictions in the DSGE-VAR $\left(\lambda=\lambda^{\hat{o p t}}\right)$, the demand shock contributes slightly less to output growth. When requiring expectations in the DSGE model to be formed according to a small-scale VAR model, the resulting share is strikingly smaller. The demand shock accounts for only $8.3 \%$ of variance in output growth, while an expectational shock to one-period ahead output growth accounts for $86 \%$.

\footnotetext{
${ }^{8}$ The posterior means for the $a, b, c$, coefficients in the lab expectations estimation (which are not shown in Table 2 to save space) are as follows: 0.31 for $a_{x}$ and 0.20 for $a_{\pi}, 0.55$ for $b_{x}$ and 0.20 for $b_{\pi}, 0.25$ for both $c_{x}$ and $c_{i}$; the data are hence uninformative for $c_{x}$ and $c_{i}$, but informative for the other coefficients.
} 
Expectational shocks also explain $46 \%$ of inflation fluctuations and $48 \%$ of interest rate fluctuations. Those shares are, instead, captured by cost-push shocks (for the case of inflation) and by demand shocks (for the case of interest rates) in the estimated model under RE.

\section{Conclusions}

Monetary policymakers increasingly rely on their ability to influence private-sector expectations, by exploiting the so-called expectations channel of monetary policy, in their efforts to stabilize the economy.

We have shown, in this paper, that the leading model for the study of monetary policy largely fails in matching the dynamic comovements between macroeconomic realizations and macroeconomic expectations that seem to exist in the data. The failure appears in large part due to the assumption of rational expectations. When we relax rational expectations to include, at least, shares of agents that form non-fully-rational expectations, based on VAR/AR models, on lab evidence, and which may be heterogeneous, the model's performance improves considerably. Another key feature in matching the data seems the inclusion of sentiment or expectation shocks, as sources of aggregate fluctuations, in addition to typical fundamental disturbances.

But even our specifications with alternative expectation formation schemes remain far from fitting the joint evolution of macroeconomic variables and expectations as well as the more flexible best-fitting DSGE-VARs. In our backward-looking expectation formation models, we have assumed constant parameters. Adding time-variation through learning may improve the ability of the model to explain the expectations data. Understanding the directions in which to extend the model to successfully capture the co-movement between macroeconomic variables and expectations remains a priority for future research.

\section{References}

[1] Adolfson, M., Lasèen, Lindè, J., and M. Villani (2008). "Evaluating an Estimated New Keynesian Small Open Economy Model", Journal of Economic Dynamics and Control, 32(8), 2690-2721.

[2] Assenza, T., Brock, W.A., and C.H. Hommes, (2012). "Animal Spirits, Heterogeneous Expectationsand the Amplification and Duration of Crises", CeNDEF Working Paper, University of Amsterdam. 
[3] Bernanke, B.S., (2004). "The Logic of Monetary Policy," remarks before the National Economists Club, Washington, D.C, December 2.

[4] Blinder, A.S., Ehrmann, M., Fratzscher, M., De Haan, J., and D.J. Jansen. (2008) "Central Bank Communication and Monetary Policy: a Survey of Theory and Evidence." Journal of Economic Literature, 46(4), 910-945.

[5] Branch, W.A., and B. McGough, (2009). "A New Keynesian Model with Heterogeneous Expectations," Journal of Economic Dynamics and Control, 33(5), 1036-1051.

[6] Bullard, J., Evans, G. and Honkapohja, S. (2008). 'Monetary policy, judgment and nearrational exuberance', American Economic Review, vol. 98, pp. 1163-77.

[7] Christiano, L.J., Eichenbaum, M., and C.L. Evans, 2005. "Nominal Rigidities and the Dynamic Effects of a Shock to Monetary Policy," Journal of Political Economy 113, 1-45.

[8] Del Negro, M., Giannoni, M., and C. Patterson, (2012). "The Forward Guidance Puzzle," Staff Reports 574, Federal Reserve Bank of New York.

[9] Del Negro, M., and F. Schorfheide, (2004). "Priors from General Equilibrium Models for VARS," International Economic Review, 45(2), 643-673.

[10] Del Negro, M., and F. Schorfheide, (2005). "Policy Predictions if the Model Does Not Fit," Journal of the European Economic Association, 3(2-3), 434-443.

[11] Del Negro, M., and F. Schorfheide, (2009). "Monetary Policy Analysis with Potentially Misspecified Models," American Economic Review, 99(4), 1415-50.

[12] Del Negro, M., Schorfheide, F., Smets, F., and R. Wouters, (2007). "On the Fit of New Keynesian Models," Journal of Business and Economic Statistics, 25, 123-143.

[13] Eusepi, S., and B. Preston, 2011. "Expectations, Learning, and Business Cycle Fluctuations," American Economic Review, 101(6), 2844-72.

[14] Evans, G. W. and Honkapohja, S. (2001). Learning and Expectations in Economics. Princeton: Princeton University Press.

[15] Giannoni, M., and M. Woodford, (2003). "Optimal Inflation-Targeting Rules," NBER Chapters, in: The Inflation-Targeting Debate, pages 93-172. 
[16] Granziera, E., (2012). "Assessing the Importance of Learning in an Empirical. Monetary Model for the U.S.", mimeo, Bank of Canada.

[17] Hommes, C.H., (2011). "The Heterogeneous Expectations Hypothesis: Some Evidence from the Lab," Journal of Economic Dynamics and Control, 35, 1-24.

[18] Milani, F., 2007. "Expectations, Learning and Macroeconomic Persistence", Journal of Monetary Economics, 54(7), 2065-2082.

[19] Milani, F., 2011. "Expectation Shocks and Learning as Drivers of the Business Cycle", Economic Journal, 121(552), 379-401.

[20] Milani, F., 2013. "Sentiment and the U.S. Business Cycle", mimeo, University of California, Irvine.

[21] Sims, C.A., 2000. "Solving Linear Rational Expectations Models", Computational Economics, 20, 1-20.

[22] Svensson, L.E.O., (2004). "Challenges for Monetary Policy," paper presented at the Bellagio Group Meeting, National Bank of Belgium, Brussels, 26-27 January.

[23] Woodford, M., (2003). Interest and Prices: Foundations of a Theory of Monetary Policy. Princeton: Princeton University Press. 
Table 1: Prior \& Posterior Estimates

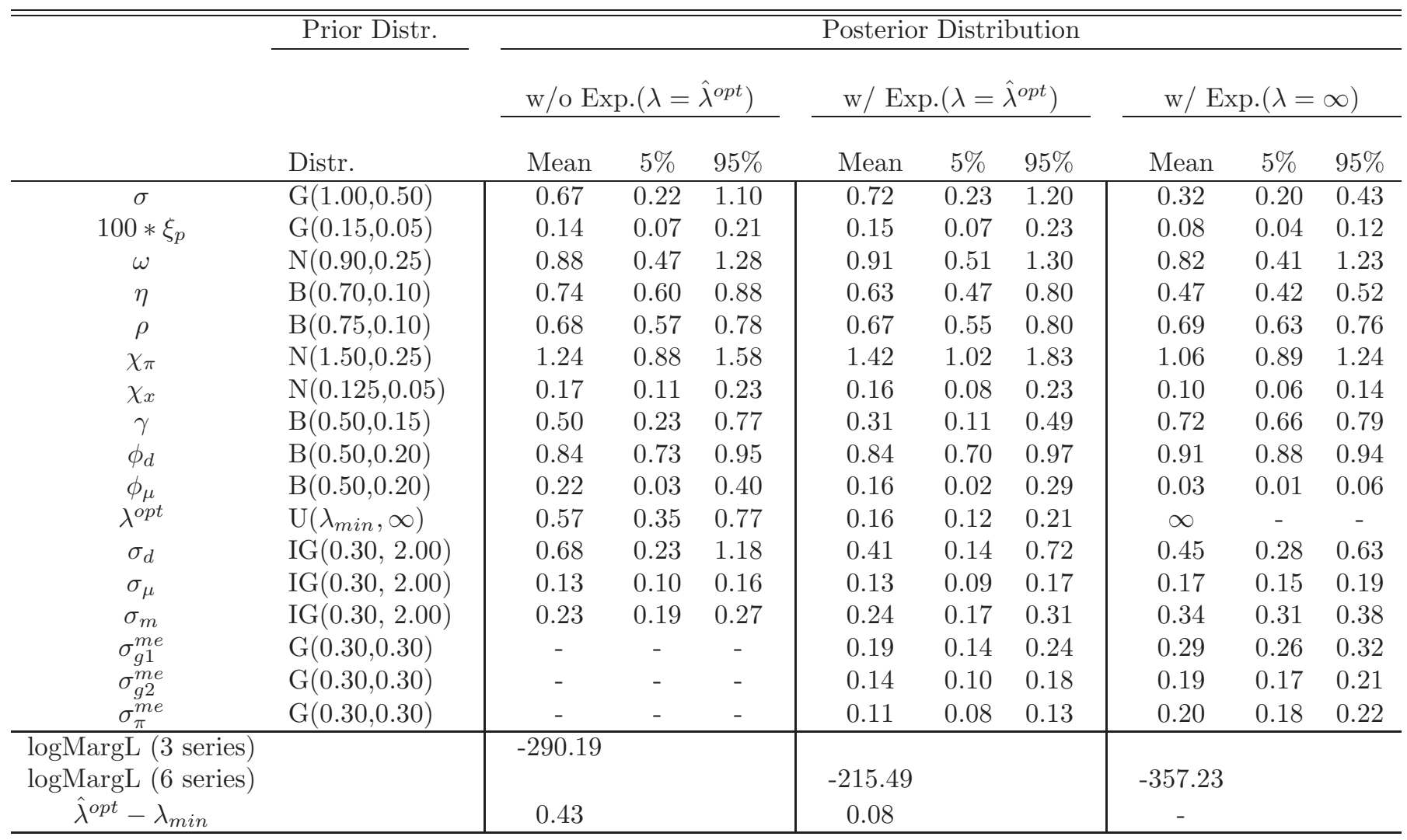

Note: w/o Exp. $(\hat{\lambda})$ correponds to DSGE-VAR $(\hat{\lambda})$ model without expectation data; w/ Exp. $(\hat{\lambda})$ corresponds to DSGE$\operatorname{VAR}(\hat{\lambda})$ model with expectation data; w/ Exp. $(\infty)$ corresponds to DSGE-VAR $(\infty)$ model with expectation data. G: Gamma Distribution, N: Normal Distribution, B: Beta Distribution, U: Uniform Distribution, IG: Inverse-Gamma Distribution 
Table 2: Prior \& Posterior Estimates with Alternative Expectations

\begin{tabular}{|c|c|c|c|c|c|c|c|c|c|c|c|c|c|}
\hline & \multirow{3}{*}{$\begin{array}{l}\text { Prior } \\
\\
\text { Distr. }\end{array}$} & \multicolumn{12}{|c|}{ Posterior Distribution } \\
\hline & & \multicolumn{3}{|c|}{ VAR } & \multicolumn{3}{|c|}{$\mathrm{AR}$} & \multicolumn{3}{|c|}{$\mathrm{HE}$} & \multicolumn{3}{|c|}{ Lab } \\
\hline & & Mean & $5 \%$ & $95 \%$ & Mean & $5 \%$ & $95 \%$ & Mean & $5 \%$ & $95 \%$ & Mean & $5 \%$ & $95 \%$ \\
\hline$\sigma$ & $\mathrm{G}(1.00,0.50)$ & 0.55 & 0.21 & 0.90 & 0.63 & 0.19 & 1.09 & 0.62 & 0.22 & 1.00 & 0.69 & 0.18 & 1.16 \\
\hline $100 * \xi_{p}$ & $\mathrm{G}(0.15,0.05)$ & 0.15 & 0.07 & 0.23 & 0.15 & 0.07 & 0.23 & 0.15 & 0.07 & 0.22 & 0.15 & 0.07 & 0.22 \\
\hline$\omega$ & $\mathrm{N}(0.90,0.25)$ & 0.89 & 0.49 & 1.31 & 0.90 & 0.49 & 1.31 & 0.89 & 0.47 & 1.30 & 0.90 & 0.50 & 1.31 \\
\hline$\eta$ & $\mathrm{B}(0.70,0.10)$ & 0.63 & 0.47 & 0.80 & 0.63 & 0.46 & 0.81 & 0.69 & 0.54 & 0.85 & 0.69 & 0.53 & 0.86 \\
\hline$\rho$ & $\mathrm{B}(0.75,0.10)$ & 0.80 & 0.71 & 0.90 & 0.78 & 0.69 & 0.89 & 0.79 & 0.70 & 0.89 & 0.79 & 0.69 & 0.90 \\
\hline$\chi_{\pi}$ & $\mathrm{N}(1.50,0.25)$ & 1.29 & 0.89 & 1.68 & 1.31 & 0.92 & 1.70 & 1.27 & 0.88 & 1.67 & 1.23 & 0.88 & 1.58 \\
\hline$\chi_{x}$ & $\mathrm{~N}(0.125,0.05)$ & 0.11 & 0.03 & 0.19 & 0.14 & 0.06 & 0.21 & 0.11 & 0.03 & 0.19 & 0.14 & 0.07 & 0.22 \\
\hline$\gamma$ & $\mathrm{B}(0.50,0.15)$ & 0.36 & 0.14 & 0.58 & 0.36 & 0.14 & 0.57 & 0.40 & 0.17 & 0.64 & 0.45 & 0.21 & 0.70 \\
\hline$\phi_{d}$ & $\mathrm{~B}(0.50,0.20)$ & 0.24 & 0.08 & 0.39 & 0.24 & 0.09 & 0.40 & 0.23 & 0.08 & 0.38 & 0.25 & 0.10 & 0.40 \\
\hline$\phi_{\mu}$ & $\mathrm{B}(0.50,0.20)$ & 0.14 & 0.02 & 0.24 & 0.14 & 0.02 & 0.24 & 0.12 & 0.02 & 0.21 & 0.11 & 0.02 & 0.21 \\
\hline$\phi_{e_{x 1}}$ & $\mathrm{~B}(0.50,0.20)$ & 0.51 & 0.29 & 0.73 & 0.50 & 0.29 & 0.70 & 0.57 & 0.36 & 0.78 & 0.54 & 0.31 & 0.78 \\
\hline$\phi_{e_{x 2}}$ & $\mathrm{~B}(0.50,0.20)$ & 0.62 & 0.47 & 0.77 & 0.63 & 0.48 & 0.77 & 0.58 & 0.43 & 0.75 & 0.56 & 0.40 & 0.72 \\
\hline$\phi_{e_{\pi}}$ & $\mathrm{B}(0.50,0.20)$ & 0.58 & 0.33 & 0.89 & 0.79 & 0.58 & 0.97 & 0.55 & 0.29 & 0.82 & 0.46 & 0.27 & 0.65 \\
\hline$\lambda$ & $\mathrm{U}\left(\lambda_{\min }, \infty\right)$ & 0.80 & 0.65 & 0.95 & 0.81 & 0.66 & 0.96 & 0.80 & 0.65 & 0.95 & 0.80 & 0.65 & 0.94 \\
\hline$\sigma_{d}$ & $\operatorname{IG}(0.30,2.00)$ & 1.71 & 0.66 & 2.67 & 1.60 & 0.67 & 2.52 & 2.03 & 0.88 & 3.17 & 2.02 & 0.72 & 3.33 \\
\hline$\sigma_{\mu}$ & $\mathrm{IG}(0.30,2.00)$ & 0.19 & 0.16 & 0.22 & 0.19 & 0.16 & 0.22 & 0.19 & 0.16 & 0.22 & 0.19 & 0.16 & 0.21 \\
\hline$\sigma_{m}$ & $\mathrm{IG}(0.30,2.00)$ & 0.21 & 0.18 & 0.25 & 0.21 & 0.18 & 0.24 & 0.21 & 0.18 & 0.25 & 0.21 & 0.18 & 0.25 \\
\hline$\sigma_{e_{x 1}}$ & $\operatorname{IG}(0.30,2.00)$ & 0.64 & 0.54 & 0.74 & 0.64 & 0.55 & 0.74 & 0.41 & 0.26 & 0.56 & 0.39 & 0.25 & 0.52 \\
\hline$\sigma_{e_{x 2}}$ & $\mathrm{IG}(0.30,2.00)$ & 0.17 & 0.14 & 0.19 & 0.17 & 0.15 & 0.20 & 0.17 & 0.14 & 0.19 & 0.16 & 0.14 & 0.19 \\
\hline$\sigma_{e_{\pi}}$ & $\mathrm{IG}(0.30,2.00)$ & 0.11 & 0.09 & 0.12 & 0.11 & 0.09 & 0.13 & 0.10 & 0.08 & 0.11 & 0.09 & 0.08 & 0.11 \\
\hline$\omega_{V A R, x}$ & $\mathrm{~B}(0.5,0.2)$ & & & & & & & 0.62 & 0.40 & 0.85 & & & \\
\hline$\omega_{V A R, \pi}$ & $\mathrm{B}(0.5,0.2)$ & & & & & & & 0.86 & 0.76 & 0.96 & & & \\
\hline$\omega_{T F, x}$ & $\mathrm{~B}(0.167,0.1)$ & & & & & & & & & & 0.20 & 0.03 & 0.36 \\
\hline$\omega_{A D, x}$ & $\mathrm{~B}(0.167,0.1)$ & & & & & & & & & & 0.27 & 0.10 & 0.44 \\
\hline$\omega_{A A, x}$ & $\mathrm{~B}(0.167,0.1)$ & & & & & & & & & & 0.13 & 0.03 & 0.22 \\
\hline$\omega_{T F, \pi}$ & $\mathrm{B}(0.167,0.1)$ & & & & & & & & & & 0.13 & 0.02 & 0.24 \\
\hline$\omega_{A D, \pi}$ & $\mathrm{B}(0.167,0.1)$ & & & & & & & & & & 0.60 & 0.48 & 0.73 \\
\hline$\omega_{A A, \pi}$ & $\mathrm{B}(0.167,0.1)$ & & & & & & & & & & 0.06 & 0.01 & 0.11 \\
\hline $\log M \operatorname{argL}$ & & -193.17 & & & -189.46 & & & -194.51 & & & -195.33 & & \\
\hline$\hat{\lambda}^{o p t}-\lambda_{\min }$ & & 0.53 & & & 0.54 & & & 0.53 & & & 0.53 & & \\
\hline
\end{tabular}

Note: VAR corresponds to the DSGE-VAR $(\hat{\lambda})$ model with expectations from a VAR that coincides with the MSV solution of the system; AR corresponds to the DSGE-VAR $(\hat{\lambda})$ model with expectations from a AR(1) model; HE corresponds to DSGE-VAR $(\hat{\lambda})$ model with heterogeneous expectations; Lab corresponds to the DSGE-VAR $(\hat{\lambda})$ model with expectations from a laboratory setting. G: Gamma Distribution, N: Normal Distribution, B: Beta Distribution, U: Uniform Distribution, IG: Inverse-Gamma Distribution 


\begin{tabular}{cccc|ccc|c}
\hline Shock to: & $r_{t}^{n}$ & $\mu_{t}$ & $\varepsilon_{t}$ & $e_{t}^{x_{1}}$ & $e_{t}^{x_{2}}$ & $e_{t}^{\pi}$ & $o_{t}$ \\
DGSE w/RE & & & & & & & \\
\hline$g_{t}$ & 93.6 & 1 & 5.4 & - & - & - & - \\
$\pi_{t}$ & 27.8 & 72.1 & 0.1 & - & - & - & - \\
$i_{t}$ & 50.1 & 15 & 34.9 & - & - & - & - \\
$E_{t} g_{t+1}$ & 61.5 & 2 & 3.4 & - & - & - & 33 \\
$E_{t} \pi_{t+1}$ & 33.5 & 47.7 & 0.1 & - & - & - & 18.8 \\
\hline Best-fitting DGSE-VAR w/RE & & & & & & & \\
\hline$g_{t}$ & 86.8 & 3.2 & 10 & - & - & - & - \\
$\pi_{t}$ & 6.3 & 93.6 & 0.1 & - & - & - & - \\
$i_{t}$ & 54.7 & 10.2 & 35.2 & - & - & - & - \\
$E_{t} g_{t+1}$ & 61.7 & 3.5 & 6.5 & - & - & - & 28.3 \\
$E_{t} \pi_{t+1}$ & 11.5 & 32.2 & 0.1 & - & - & - & 56.2 \\
\hline$g_{t}$ & & & & & & & \\
$\pi_{t}$ & 8.3 & 3.2 & 0.3 & 86 & 1.1 & 1.1 & - \\
$i_{t}$ & 1.9 & 51.6 & 0.1 & 28.1 & 0.7 & 17.7 & - \\
$E_{t} g_{t+1}$ & 2.8 & 10.6 & 38.2 & 41.7 & 1 & 5.8 & - \\
$E_{t} \pi_{t+1}$ & 33.9 & 4 & 2.6 & 53.9 & 5.2 & 0.6 & - \\
\hline
\end{tabular}

Table 3 - Variance Decomposition.

Note: The table shows the share of the forecast error variance in output growth, inflation, interest rate, expected output growth, and expected inflation, due to each structural shock (natural rate, cost-push, monetary policy), expectational shock (to expected output, one and two quarters ahead, and expected inflation), and the respective measurement error $\left(o_{t}\right)$. The results are compared across the benchmark DSGE model with RE, the best-fitting DSGEVAR under RE (with $\lambda=\hat{\lambda}^{o p t}$ ), and the DSGE-VAR model with expectations formed from small-scale VAR models, allowing for expectation shocks. 

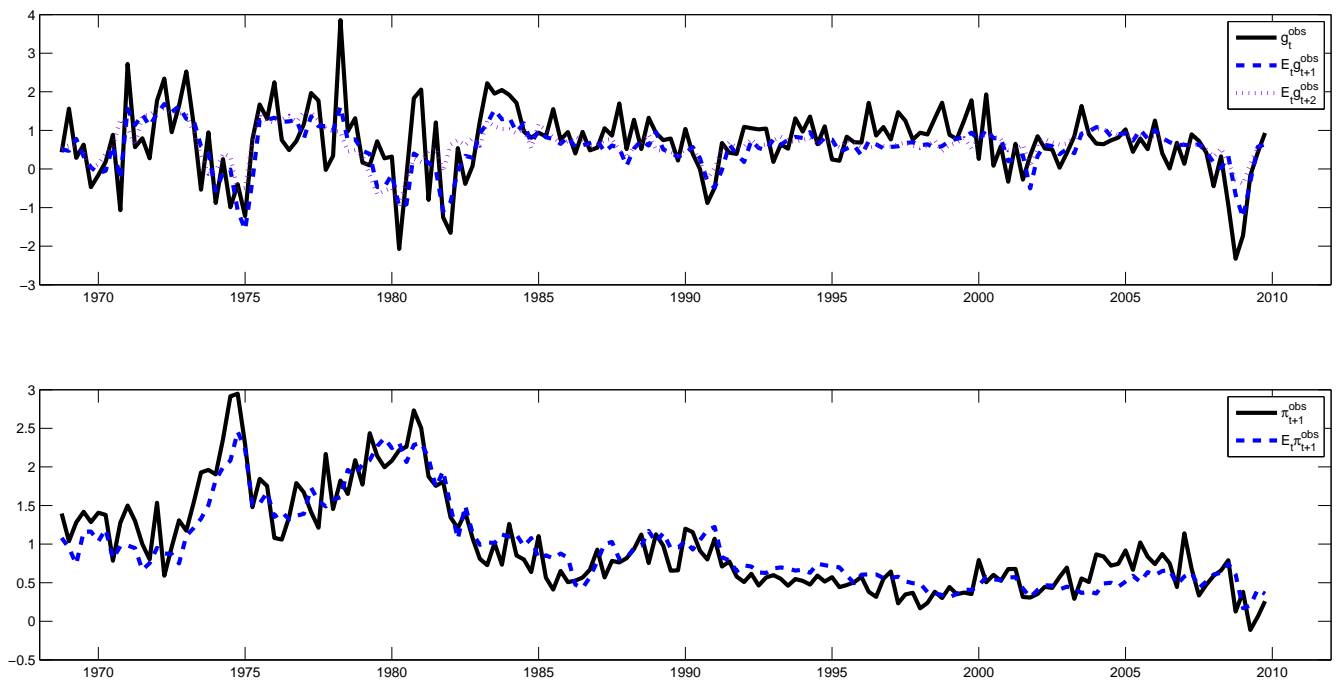

Figure 1: Real Output Growth and Inflation: Expectations and Realizations. 
IRF to Natural Rate Shock
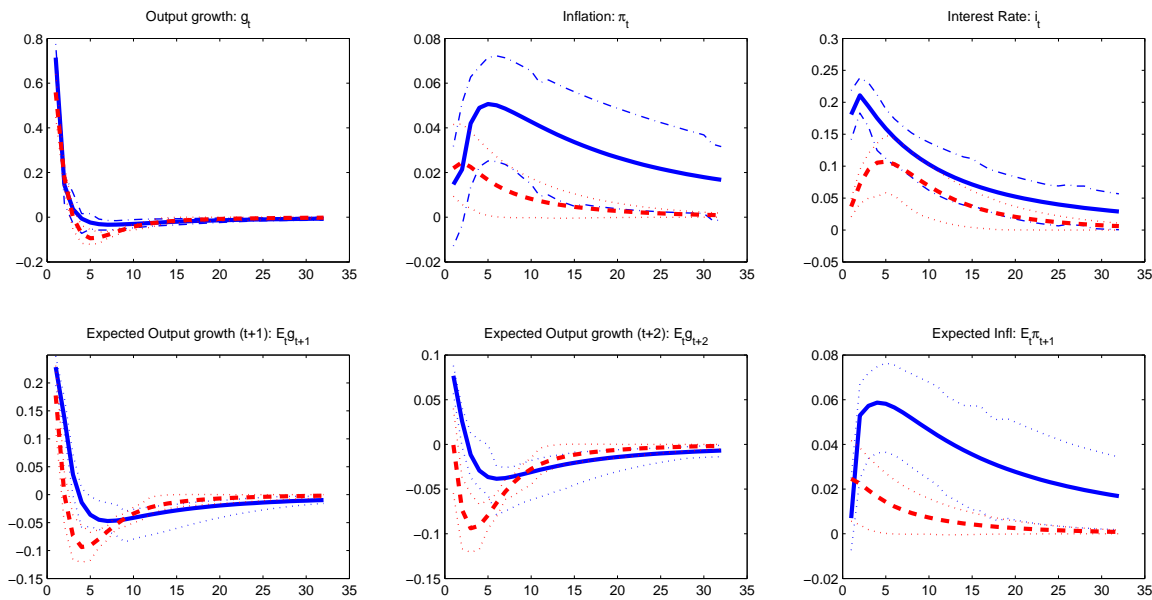

Figure 2: Impulse Response Functions. Response of observables to structural shocks. The solid blue line denotes responses obtained for the DSGE-VAR $\left(\lambda=\hat{\lambda}^{\text {opt }}\right)$ model; the dashed red line denotes responses obtained for the corresponding DSGE model. Dotted lines denote $95 \%$ error bands. 
IRF to Cost-Push Shock
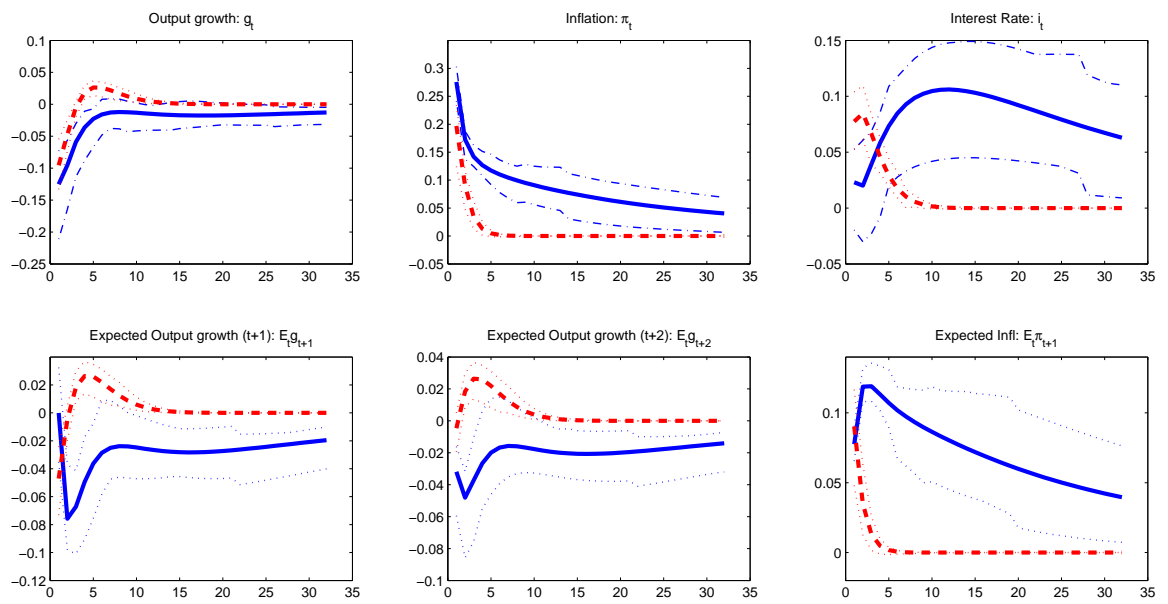

Figure 3: Impulse Response Functions. Response of observables to structural shocks. The solid blue line denotes responses obtained for the DSGE-VAR $\left(\lambda=\hat{\lambda}^{\text {opt }}\right)$ model; the dashed red line denotes responses obtained for the corresponding DSGE model. Dotted lines denote $95 \%$ error bands. 
IRF to MP Shock
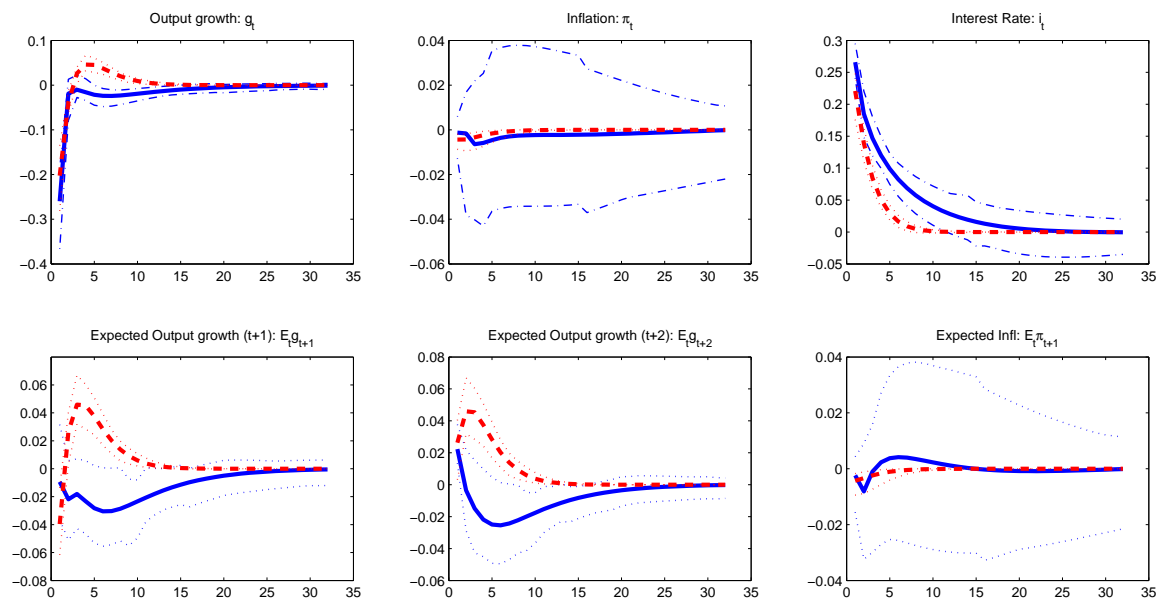

Figure 4: Impulse Response Functions. Response of observables to structural shocks. The solid blue line denotes responses obtained for the DSGE-VAR $\left(\lambda=\hat{\lambda}^{\text {opt }}\right)$ model; the dashed red line denotes responses obtained for the corresponding DSGE model. Dotted lines denote $95 \%$ error bands. 